\title{
Grundkonzepte der gastrointestinalen Psychophysiologie
}

\author{
Ulrich Cuntz \\ Schön Klinik Roseneck, Prien am Chiemsee, Deutschland
}

Schlüsselwörter

Reizdarmsyndrom · Psychophysiologie .

Gastrointestinale Motilität - Mikrobiom · Psychischer Stress

\section{Zusammenfassung}

Die wissenschaftliche Beschäftigung mit der Psychophysiologie des Gastrointestinaltrakts hat mittlerweile eine lange Tradition und hat zu beachtlichen Ergebnissen geführt. Dabei haben sich die Grundkonzepte und Forschungsansätze in den letzten 50 Jahren erheblich gewandelt, weisen dabei aber doch eine bemerkenswerte Konvergenz auf. Der Gastrointestinaltrakt reagiert auf unterschiedliche Stressoren mit unterschiedlichen Motilitätsmustern; verschiedene Abschnitte des Gastrointestinaltrakts können dabei in unterschiedlicher, gegebenenfalls auch gegensinniger Weise reagieren. Es gibt Hinweise, aber keine zweifelsfreien Belege dafür, dass unterschiedliche Emotionen mit unterschiedlichen Reaktionsmustern assoziiert sind. Entgegen der ursprünglichen Annahme gibt es keinen eindeutigen Zusammenhang zwischen Motilitätsmustern und gastrointestinalen Beschwerden. Das Reizdarmsymdrom, die funktionelle gastrointestinale Störung mit der größten klinischen Bedeutung, ist mit einer intestinalen Hyperalgesie, Hypersensitivität und Hypervigilanz assoziiert. Dies hängt unmittelbar mit der zentralnervösen Reizverarbeitung zusammen: Bei Reizdarmpatienten werden bei intestinalen Schmerzreizen vermehrt Kerngebiete aktiviert, die mit der Schmerzwahrnehmung und der Stressverarbeitung zusammenhängen. Es lässt sich auch zeigen, dass an der viszeralen Hyperalgesie die Hypothalamus-Hypophysen-Nebennierenrinden (HPA)-Achse und insbesondere die Corticotropin-Releasing-Factor-1 (CRF1)-Rezeptoren beteiligt sind. Entgegen früheren Annahmen sind auch immunologische Prozesse beim Reizdarmsyndrom involviert, und auch diese sind von psychologischen Faktoren abhängig. Stress nimmt über verschiedene Wege Einfluss auf die immunologische Reaktion. Diese Entzündungsvorgänge entstehen dabei in Auseinandersetzung mit der bakteriellen Besiedelung des Darms, insbesondere des Colons. Stress nimmt Einfluss auf die qualitative und quantitative Zusammensetzung unseres "Mikrobioms", und die Zusammensetzung des Mikrobioms moderiert wiederum die Stressantwort. Offensichtlich erfordert ein vollständiges Verständnis funktioneller gastorintestinaler Beschwerden auch die Einbeziehung des Mikrobioms und der damit verbundenen immunologischen Vorgänge.

\author{
Keywords \\ Irritable bowel syndrome · Psychophysiology . \\ Gastrointestinal motility · Microbiome - Psychological stress
}

\section{Summary}

Basic Concepts of Gastrointestinal Psychophysiology

By now, scientific research on the psychophysiology of the gastrointestinal tract has a long tradition and has led to remarkable results. The basic concepts and research approaches have changed considerably during the last 50 years, but still show a clear convergence. The gastrointestinal tract reacts to different stressors with different motility patterns; different sections of the gastrointestinal tract can hereby react in different, sometimes opposing ways. There are hints, though no clear-cut evidence, in favor of the notion that different emotions are associated with different reaction patterns. In contrast to the original assumption, there is no clear relationship between the motility patterns and the various kinds of gastrointestinal discomfort. The irritable bowel syndrome, as the functional gastrointestinal disorder with the highest clinical significance, is associated with intestinal hyperalgesia, hypersensitivity, and hypervigilance. This is directly related to the processing of stimuli in the central nervous system: In patients with irritable bowel syndrome, intestinal pain stimuli lead to increased activation of nuclear areas related to pain perception and stress processing. It has also been shown that the hypothalamic-pituitary-adrenal (HPA) axis and especially the corticotropin-releasing factor 1 (CRF1) receptors are involved in visceral hyperalgesia. In contrast to earlier assumptions, immunological processes are involved in the irritable bowel syndrome as well, and these processes are also dependent on psychological factors. Stress affects the immunological reactions via diverse pathways. These inflammatory processes originate from struggling with the bacterial colonization of the intestine, and especially of the colon. Stress influences the qualitative and quantitative composition of our 'microbiome', and the microbiome composition in turn modulates the stress response. Obviously, a full understanding of the functional gastrointestinal disorders also requires consideration of the microbiome and its related immunological processes.

\begin{tabular}{ll}
\hline KARGER & (1) 2014 S. Karger GmbH, Freiburg \\
1016-6262/14/0242-0100\$39.50/0 \\
$\begin{array}{l}\text { Fax +49 7614520714 } \\
\text { Information@Karger.com } \\
\text { www.karger.com }\end{array}$ & $\begin{array}{l}\text { Accessible online at: } \\
\text { www.karger.com/ver }\end{array}$
\end{tabular}




\section{Einleitung}

Kaum ein anderer Bereich der Psychosomatik und der Körper-Seele-Zusammenhänge ist bereits im vorwissenschaftlichen Verständnis von so hohen und weitgehenden Erwartungen belastet wie das Zusammenspiel zwischen Psyche und Gastrointestinaltrakt. Sei es das Gefühl, das im Bauch gespürt wird, die Angst, die einem den Magen zuschnürt, die Wut, die man im Bauch hat, oder der «Schiss», den man vor belastenden Situationen hat; wir sind offensichtlich von dem besonderen Verhältnis zwischen Gefühlen und den Empfindungen und Funktionsänderungen im Gastrointestinaltrakt überzeugt.

Somit ist es kein Wunder, dass die Forschung diese vorwissenschaftlichen Überzeugungen aufgenommen und zu weitreichenden Forschungshypothesen entwickelt hat. Dabei ging und geht es aus Sicht der Psychosomatik im Wesentlichen darum, gastroenterologische Erkrankungen und Funktionsstörungen über psychophysiologische Mechanismen zu erklären. So formulierten Mirsky et al. [1957] in den 1950er-Jahren ihre somatopsychisch-psychosomatische Genese des Ulcus duodeni. Dementsprechend soll ein hoher Pepsinspiegel in der frühen Kindheit zu einer vermehrten Abhängigkeit von der nährenden Mutter führen, die im späteren Leben in Anbetracht von Dependenzkonflikten zum Auftreten von Ulcera duodeni führt. Engel [1952, 1954a,b] nahm in Anlehnung an die «Spezifitätshypothese» von Alexander [1985] an, dass die Colitis ulcerosa eine spezifische Psychogenese aufweise, d.h. dass es typische Konfliktsituationen in der Kindheit und Jugend von Colitis-ulcerosa-Patienten gäbe, die dann im Erwachsenenalter zur manifesten Erkrankung führten. Noch in den 1970erJahren postulierten Hislop [1971] und Latimer [1979], dass das Reizdarmsyndrom nichts anderes sei als eine larvierte Depression. Keine dieser Annahmen ist heute noch haltbar, und trotzdem haben sie die Forschung beeinflusst, befruchtet und am Ende erheblich weitergebracht. Die Psychosomatik des Gastrointestinaltrakts, die wir heute anhand wissenschaftlicher Befunde beschreiben können, sieht anders aus, als es die naiven Vorannahmen erahnen ließen. Der vorliegende Beitrag soll die basalen Grundkonzepte und Forschungsparadigmata skizzieren, die in den letzten 50 Jahren die Forschung vorangetrieben haben. Ein wesentliches Ziel der in den letzten Jahren immer intensiver werdenden Forschung war und ist es dabei, die Pathogenese der häufigen funktionellen Störungen des Gastrointestinaltrakts, wie z.B. des Reizdarmsyndroms, aufzuklären und damit einen Beitrag zu therapeutischen Ansätzen zu leisten. Darüber hinaus besteht die Vermutung, dass chronische organische Erkrankungen des Gastrointestinaltrakts hier spielen immer noch die chronisch-entzündlichen Darmerkrankungen die größte Rolle - in Aktivität und Verlauf ebenso von psychosozialen Einflüssen berührt werden. Bemerkenswert ist, dass sich im Laufe der Jahre die Grundvorstellungen davon, wie psychische Prozesse Einfluss auf die primär autonome Funktion des Gastrointestinaltrakts nehmen, erheblich gewandelt haben. War am Anfang vorwiegend die Beeinflus- sung der Motilität im Fokus der Forschung, weitete sich das Spektrum auf die gesamte «Brain-Gut-Achse» aus, wobei insbesondere die sensiblen Funktionen von Interesse waren und sind. Neuere Forschungsansätze betreffen inflammatorische und immunologische Vorgänge sowie in den letzten Jahren auch die direkte immunologische Auseinandersetzung des Körpers mit den Darmbakterien.

\section{Motilität}

Bis in die Mitte der 1990er-Jahre stand die Erforschung der gastrointestinalen Motilität ganz im Zentrum psychophysiologischer Forschung. Dabei lassen sich implizit verschiedene Grundannahmen formulieren, die Voraussetzung dieses Forschungsansatzes waren und sind:

- Psychosoziale Stressoren verändern den Funktionszustand der gastrointestinalen Motilität.

- Emotionen haben einen spezifischen Effekt auf die Motilität.

- Störungen der gastrointestinalen Motilität führen zu den typischen Beschwerden, die funktionelle Störungen charakterisieren,

- dabei führen unterschiedliche emotionale Zustände auch zu unterschiedlichen Änderungen der Motilität und damit $\mathrm{zu}$ unterschiedlichen Beschwerdemustern. Bereits in den späten 1940er-Jahren hatte die New Yorker Forschungsgruppe um Thomas P. Almy [Almy et al., 1949; Almy, 1951] umfangreiche Untersuchungen zur gastrointestinalen Motilität unter dem Einfluss von psychosozialer Belastung und induzierten Emotionen durchgeführt. Dabei wurden einfache Ballonkatheter sowie die endoskopische Beurteilung der Motilität verwendet. Nach wissenschaftlichen Kriterien sind diese Forschungsergebnisse angreifbar: Es lag kein streng experimentelles Design vor, und die Untersuchungsbedingungen mit Ballonkatheter und Endoskopie dürften an sich schon als Stressoren betrachtet werden, die selbst einen Einfluss auf die Motilität hatten. Emotionale Zustände wurden in Interviews induziert, bei denen über belastende Lebensereignisse geredet wurde. Trotz dieser Einschränkungen waren die Ergebnisse dieser Forschungsgruppe von großem Einfluss auf die Forschung der folgenden Jahre. Vor allem bei der Subgruppe der Patienten, die nach heutiger Terminologie unter einem Reizdarmsyndrom vom Diarrhötyp klagten, waren die in den Interviews vorgefundenen Gefühlsregungen vor allem durch Angst und Hilflosigkeit geprägt. Wenn diese Emotionen auftraten, fanden die Forscher gleichzeitig einen Tonusverlust und ein Nachlassen der Kontraktionswellen im Sigma. Die daraus deduzierte Annahme, die sich später verifizieren ließ, lautet, dass funktionelle Diarrhö durch ein Nachlassen der kontraktilen Aktivität des Colons charakterisiert ist. Angst und Hilflosigkeit führen somit über einen Tonusverlust des Colons zur Diarrhö. 
Bei Patienten mit einem obstipationsbetonten Subtyp des Reizdarmsyndroms traten in den Interviews vorwiegend die Emotionen Ärger und Wut auf. Hierbei fanden sich gleichzeitig ein Anstieg des Colontonus und eine Intensivierung der Colonmotilität. Obstipation sei dementsprechend durch eine Intensivierung der kontraktilen Aktivität des Colons charakterisiert.

In diesen Ergebnissen finden sich alle oben genannten Grundannahmen zur Motilität wieder: Emotionen beeinflussen die gastrointestinale Motilität, wobei die Qualität der Emotionen für die Art der Funktionsänderung entscheidend ist. Diese wiederum entscheidet über die Art der auftretenden Beschwerden. In den folgenden Jahren ließ sich ein Teil dieser Annahmen belegen; der Rest kann zumindest als bislang unwiderlegt, letztlich wohl aber auch als unwiderlegbar gelten.

Die Hypothese, dass funktionelle Diarrhö durch einen Tonusverlust im Colon charakterisiert ist, ließ sich in späteren Untersuchungen [Connell, 1962; Wangel et al., 1965] belegen. Weniger gesichert ist die Annahme, dass Obstipation durch eine vermehrte motorische Aktivität des Colons verursacht wird.

Einen indirekten Beleg für den differenziellen Einfluss von Emotionen auf die Colonmotilität lieferten Gorard et al. [1996], indem sie die Colontransitzeit bei Angstpatienten, depressiven Patienten und normalen Kontrollpersonen verglichen. Dabei war der Colontransit bei Angstpatienten (ohne dass diese über Diarrhö geklagt hätten) signifikant verkürzt und bei depressiven Patienten signifikant, aber bei fehlender subjektiver Symptomatik gegenüber Kontrollen verlängert.

Welgan et al. [1985, 1988] induzierten bei Kontrollpersonen und Reizdarmpatienten in einer raffiniert angeordneten experimentellen Situation Ärger und Angst und konnten den erwarteten Befund erheben: Die myoelektrische Aktivität stieg unter den Stressbedingungen signifikant an, und zwar bei Reizdarmpatienten ausgeprägter als bei Kontrollpersonen. Gleichzeitig zeigte dieser Versuch allerdings auch die Grenzen des experimentellen Forschungsparadigmas an: Die beobachteten Effekte waren nicht die unmittelbare Folge der experimentellen Bedingungen, sondern die Folge der unterschiedlichen Wahrnehmung von Kontroll- und Experimentalbedingungen. Reizdarmpatienten ärgerten sich wesentlich mehr über die gleichen experimentellen Reize. Verbunden mit dieser deutlich stärker ausgeprägten emotionalen Reaktion zeigten Reizdarmpatienten eine ebenso signifikant stärker ausgeprägte motorische Aktivität bereits unter Ruhebedingungen. Unter den Stressbedingungen stieg sowohl bei Kontrollpersonen als auch bei den Reizdarmpatienten die motorische Aktivität signifikant an. Die gleiche Forschergruppe [Welgan et al., 2000] konnte mit einem vergleichbaren experimentellen Design zeigen, dass Ärger zwar die motorische Aktivität des Colons erhöht, gleichzeitig aber die Aktivität des Magenantrums verringert. Dies belegt, dass die Motilität der intestinalen Hohlorgane bei vergleichbaren Reizen gegensinnig reagieren kann.

Ein grundsätzliches Problem bei der Einschätzung der Bedeutung von emotionalen Einflüssen auf die gastrointestinale Motilität und hierdurch verursachte Beschwerden liegt darin, dass die gastrointestinale Motilität nicht unter Alltagsbedingungen messbar ist und dass sich Beschwerden nicht ohne weiteres in experimentellen Situationen provozieren lassen. Vergleichsweise gut messen und charakterisieren lassen sich Motilitätsstörungen der Speiseröhre. Die Speiseröhre zeigt unter Normalbedingungen eine eindeutig zum Magen gerichtete propulsive Aktivität während des Schluckakts. Störungen der Motilität können die Höhe der Druckamplitude oder die Propulsion der Kontraktionswellen betreffen. Verschiedene Formen von Stressoren wie Lärm und anspruchsvolle kognitive Aufgaben können die ösophageale Motilität so beeinflussen, dass eine erhöhte Druckamplitude auftritt [Anderson et al., 1989] oder dass beim Cold-Pressure-Test oder beim dichotomen Hören vermehrt simultane, also nichtpropulsive Ösophaguskontraktionen auftreten [Ayres et al., 1989]. Wenn also Stressexperimente zu einem vermehrten Auftreten nichtpropulsiver Wellen sowie einer erhöhten Kontraktionsamplitude führen und diese ihrerseits mit Schmerzen assoziiert sind, ließe sich hieraus ein geschlossenes psychophysiologisches Modell der Entstehung von ösophagealen Beschwerden deduzieren. Allerdings treten während dieser Stressexperimente kaum Beschwerden auf. Letztlich kommt Richter [2001] zu dem Ergebnis: «Although manometric patterns have been defined for these disorders, the relation with symptoms is poorly defined and the response to medical or surgical therapy unpredictable.» Das heißt, dass auch dann, wenn Patientengruppen mit funktionellen Störungen des Ösophagus sich durch eine gestörte Motilität charakterisieren lassen, es dennoch keinen eindeutigen Zusammenhang zwischen diesen Motilitätsphänomenen und -beschwerden gibt. Die Auffälligkeiten der Motilität sind damit eher Epiphänomene einer den Beschwerden und den Änderungen der Motilität zugrunde liegenden gemeinsamen Störung.

Aus den bereits zitierten Beobachtungen von Almy lässt sich die Hypothese ableiten, dass der Gastrointestinaltrakt (anders als das Herz-Kreislauf-System) auf unterschiedliche emotionale Reize mit unterschiedlichen Antworten reagiert. Ein Experiment von Rao et al. [1998] verwendete unterschiedliche Stressoren und verglich eher psychologisch wirksame Stressoren (dichotomes Hören) mit physischen Stressoren (ColdPressure-Test). Dabei traten im Colon unter psychologischen Stressoren signifikant mehr propagierende Kontraktionen auf, während sich dies beim Cold-Pressure-Test nur für simultane Kontraktionswellen belegen ließ. Propagierende Wellen im Colon führen zur Stuhlentleerung, während simultane Kontraktionen im Colon mit einer verzögerten Colontransitzeit assoziiert sind. Allerdings lässt sich aus diesem Experiment weder ein Bezug zu unterschiedlichen Emotionen noch zu unterschiedlichen Beschwerden folgern. Belegt wird allerdings hierdurch, dass das Colon - im Gegensatz zum Herz-KreislaufSystem - unterschiedliche Reaktionstypen aufweisen kann.

Wegen der mangelnden Assoziation von Motilitätsphänomenen mit Beschwerden wird in den letzten Jahren zunehmend weniger in diesem Bereich geforscht. Möglicherweise ergeben sich aber neue Impulse für die Motilitätsforschung aus 
neueren Erkenntnissen zu den zentralen Regulationsmechanismen der gastrointestinalen Motilität. Zentralnervöse Corticotropin-Releasing-Factor (CRF)-Signalwege sind bekanntlich in die endokrine, immunologische und viszerale Antwort auf Stress involviert. Die Identifizierung von verschiedenen Elementen des CRF-Systems, insbesondere die Darstellung der zwei CRF-Rezeptorsubtypen 1 und 2 und die gleichzeitige Verfügbarkeit von selektiven CRF1-Rezeptorantagonisten, haben zu guten experimentellen Belegen geführt, dass diese Rezeptoren in die spezifische Reaktionsweise des Gastrointestinaltrakts auf Stress involviert sind. Dabei erzeugen physische Stressoren wie Schmerzreize und körperliche Anstrengung über den CRF2-Rezeptor eine Verzögerung der Magenentleerung und eine Verminderung der Magenkontraktilität. Im Gegensatz dazu scheinen psychologische Stressoren vorwiegend über den CRF1-Rezeptor zu wirken. Solche Stressoren können konditionelle Angst, Wasservermeidung oder Restraint Stress (Fixierung von Extremitäten) im Tierexperiment sein. Diese führen zu einer Absenkung der Colontransitzeit und einer Steigerung der Colonmotilität. Die Aktivierung der zentralen CRF1-Rezeptoren hängt mit der stressbezogenen endokrinen Antwort und dem Auftreten von angstähnlichem Verhalten bei Tieren zusammen. Sowohl die zentralnervösen als auch die peripheren CRF1-Rezeptoren führen bei Nagetieren zu einer Stimulation der Motilität, des Transits, der Sekretion und der intestinalen Permeabilität des Colons. Darüber hinaus lässt sich bei diesen Tieren auch das Auftreten von Diarrhö beobachten. Diese Rezeptoren scheinen gleichzeitig auch zu einer vermehrten intestinalen Schmerzwahrnehmung zu führen. Die Motilitätsphänomene werden im Zusammenhang dieser neueren Befunde zu Teilaspekten einer neuronale, sensorische, endokrine, immunologische und motorische Reaktionen umfassenden Stressantwort [Tache et al., 2002, 2004a,b, 2005].

\section{Intestinale Sensibilität}

Der Gastrointestinaltrakt funktioniert in vielen Aspekten autonom und ist dennoch mit dem zentralen Nervensystem eng verbunden. Die wichtigste nervale Verbindung zwischen dem zentralen Nervensystem und dem Gastrointestinaltrakt ist der Nervus vagus, der zu wesentlichen Teilen sensible Fasern führt. Im Bewusstsein für seinen Körper spielt die sensible Innervierung des Magen-Darm-Trakts im Alltag für den Menschen anscheinend eine untergeordnete Rolle. Die vermehrte Wahrnehmung intestinaler Stimuli scheint aber eine wesentliche Rolle bei der Entstehung von gastrointestinalen Beschwerden zu spielen. Üblicherweise wird die gastrointestinale Sensibilität mit einem Ballonkatheter getestet, der in unterschiedlichen Teilen der intestinalen Hohlorgane platziert werden kann. Zumeist geschieht dies wegen der leichten Erreichbarkeit im Rektum. Unterschieden wird dabei zwischen Hypervigilanz (vermehrte Aufmerksamkeit), Hypersensibilität (herabgesetzte Wahrnehmungsschwelle) und Hyperalgesie (herabgesetzte Schmerzschwelle). Die erste Beschreibung von viszeraler Hypersensitivität und Hyperalgesie stammt von Ritchie [1973] und wurde bereits in den 1970er-Jahren veröffentlicht. Generell zeigen Reizdarmpatienten im Vergleich zu Kontrollpersonen sowohl eine erniedrigte Wahrnehmungsschwelle als auch eine erniedrigte Schmerzschwelle im Rektum und Sigma [Whitehead et al., 1998, 1990; Mayer und Tillisch, 2011; Mertz, 2002]. Während eine abnormal niedrige Wahrnehmungsschwelle nur bei etwa der Hälfte der Patienten gesehen wird, findet sich eine Veränderung der Wahrnehmung für viszerale Stimuli bei nahezu allen Patienten mit Reizdarmsyndrom [Mertz, 2002]. Dabei scheint Hypervigilanz gegenüber unangenehmen viszeralen Stimuli bei nahezu allen Patienten mit Reizdarmsyndrom vorzuliegen. Veränderungen der Wahrnehmungsschwelle bei kontrollierter rektosigmoidaler Dehnung kommen jedoch vor allem bei weiblichen Patienten mit einem Reizdarmsyndrom vom Obstipationstyp vor. Dagegen findet man dies kaum bei männlichen Patienten mit Reizdarmsyndrom vom Diarrhötyp [Schmulson, 1999, 2001].

Hinweise darauf, dass es sich bei der intestinalen Hyperalgesie um Veränderungen der zentralen Schmerzmodulation handelt, kommen aus der funktionellen Hirnbildgebung. Verschiedene Studien, die mit funktioneller Magnetresonanztomographie (fMRI) Kontrollpersonen und Reizdarmpatienten unter kontrollierter rektaler Ballondistension untersuchten, demonstrieren konsistent eine Aktivierung in Hirnregionen der viszeralen Reizverarbeitung. Patienten zeigten dabei eine stärker ausgeprägte Aktivierung in Hirnregionen, die mit Stress und Erregung assoziiert sind, und ausschließlich bei den Patienten waren Hirnregionen der zentralen Schmerzverarbeitung aktiviert [Tillisch et al., 2011]. Elsenbruch et al. [2010a,b] konnten zeigen, dass unter Stressbedingungen die Modulation der Aktivierung unterschiedlicher Gehirnregionen bei Reizdarmpatienten deutlich ausgeprägter war, während sie unter Entspannungsbedingungen reduziert war. Diese Ergebnisse weisen darauf hin, dass vermehrte Schmerzwahrnehmung kein auf Rezeptorebene vorgegebener Unterschied zwischen Reizdarmpatienten und Kontrollpersonen ist, sondern offensichtlich von psychischen Faktoren und Umweltbedingungen abhängig ist.

Im Tierexperiment wurde belegt, dass Stresshormone im zentralen Kern der Amygdala die Colonhypersensitivität regulieren [Johnson et al., 2012]. Dabei wird die Hypersensitivität über einen CRF1-Rezeptor-abhängigen Mechanismus gesteuert. In diesen experimentellen Studien sind bei der viszeralen Hyperalgesie periphere wie auch zentrale CRF1-Subtyprezeptoren involviert. Die Aktivierung der Subtyp-2-Rezeptoren scheint dagegen eine analgetische Wirkung zu haben. Viszerale Hypersensitivität und Hyperalgesie scheinen damit unmittelbar mit den Beschwerden des Reizdarmsyndroms zusammenzuhängen [Larauche et al., 2012]. Die experimentelle Beeinflussbarkeit und die Abhängigkeit viszeraler Hypersensitivität von einem gut beschriebenen endokrinen Mechanismus öffnen einen Weg zu einer psychotherapeutischen und letztlich auch einer pharmakologischen Beeinflussbarkeit dieser Phänomene. 


\section{Inflammation}

Die Darmwand hat die Aufgabe, Nährstoffe, Elektrolyte und Spurenelemente aufzunehmen und gleichzeitig - wie die äußere Haut - den Organismus vor dem Eindringen der im Dickdarm befindlichen Mikroorganismen zu schützen. Dies ist die Funktion der Schleimhautbarriere, die ein eigenständiger, teils genetisch determinierter, teils in Auseinandersetzung mit der Außenwelt sich ständig neu anpassender Bestandteil unseres Immunsystems ist. Für die chronisch entzündlichen Darmerkrankungen Colitis ulcerosa und Morbus Crohn gilt es mittlerweile als sehr wahrscheinlich, dass eine Fehlregulation der adaptiven Immunantwort gegen Mikroorganismen zu den Entzündungsreaktionen und den damit verbundenen Symptomen führt. Hierbei spielen sicherlich genetische Faktoren eine wichtige Rolle. Gleichzeitig scheinen aber auch Stress und belastende Lebensereignisse den Verlauf der chronisch entzündlichen Darmerkrankungen $\mathrm{zu}$ beeinflussen. In einer Übersichtsarbeit fasst Rampton [2009] das Ergebnis von 11 prospektiven Studien zur Beeinflussung der chronisch entzündlichen Darmerkrankung durch Stress zusammen: 8 dieser Studien zeigen eine signifikante Assoziation von Stress mit nachfolgender Verschlimmerung der entzündlichen Darmerkrankung. Für die Stressabhängigkeit der Immunantwort auf intestinale Mikroorganismen gibt es verschiedene, tierexperimentell belegte Erklärungsansätze:

- Sympathisches Nervensystem: Die Mastzellen der Darmmukosa stehen in engem Kontakt mit sympathischen und parasympathischen Nervenendigungen. Das sympathische Nervensystem hat aber eine entzündungsfördernde Rolle [Tache et al., 2004b].

- Parasympathisches Nervensystem: Im Gegensatz dazu hat der Nervus vagus eine entzündungshemmende Rolle. Es lässt sich zeigen, dass die Produktion proentzündlicher Zytokine durch Makrophagen über vagale Nervenendigungen gehemmt wird [Pavlov und Tracey, 2012].

- HPA-Achse: Stress erhöht die Mukosa-Permeabilität des Colons bei Ratten über einen CRF-abhängigen Mechanismus, bei dem vermehrt der entzündungsfördernde Tumornekrosefaktor (TNF) freigesetzt wird [Yuan et al., 2012].

- Letztlich erscheint auch die Mikroflora des Darms selbst stressabhängig und in der Lage zu sein, die Immunantwort zu moderieren [Bailey et al., 2011; Sudo et al., 2004] (hierauf wird im letzten Abschnitt noch einmal näher eingegangen).

Mittlerweile gibt es immer mehr Belege dafür, dass entzündliche Prozesse auch beim Reizdarmsyndrom eine wichtige Rolle spielen, obwohl makroskopisch und histologisch die Colonschleimhaut nicht entzündlich verändert ist [Gwee, 2010]. Ausgangspunkt der diesbezüglichen Forschung war die Beobachtung, dass sich nach einem akuten Magen-Darm-Infekt in sehr vielen Fällen ein chronisch verlaufendes Reizdarmsyndrom anschließt. In zahlreichen Studien war die Wahrscheinlichkeit des Auftretens eines Reizdarmsyndroms nach Infektionen des Gastrointestinaltrakts deutlich erhöht [Schwille-Kiuntke et al., 2011; Spiller und Garsed, 2009a]: Dabei liegt die Wahrschein- lichkeit eines postinfektiösen Reizdarmsyndroms in Abhängigkeit von dem jeweiligen Erreger der Gastroenteritis zwischen 7 und $36 \%$. Bereits eine der ersten Studien von Gwee et al. [Gwee et al., 1999], die dieses Phänomen beschrieb, belegte auch die Bedeutung psychologischer Faktoren für den weiteren Verlauf nach akuter Gastroenteritis. In einer prospektiven Studie untersuchten die Forscher über 90 Patienten, die wegen einer Gastroenteritis behandelt wurden. Patienten die ein persistierendes postinfektiöses Reizdarmsyndrom entwickelten, hatten zum Zeitpunkt der Gastroenteritis höhere Werte für Ängstlichkeit, Neurotizismus und Somatisierungsneigung und berichteten mehr belastende Lebensereignisse. Bei den persistierend symptomatischen Patienten war 3 Monate nach der akuten Darmentzündung eine erhöhte entzündliche Aktivität in den Darmbiopsien nachweisbar sowie eine verminderte Reizschwelle im Rektum. Das postinfektiöse Reizdarmsyndrom bietet die Möglichkeit der prospektiven Untersuchung von initial klinisch unauffälligen Patienten. In einer Übersichtsarbeit über verschiedene Studien beziffern Spiller und Garsed [2009b] die Wahrscheinlichkeit des Auftretens eines postinfektiösen Reizdarmsyndroms in Abhängigkeit von vorliegenden belastenden Lebensereignissen als 2-fach erhöht, in Abhängigkeit von vorliegender Hypochondrie ebenfalls als 2-fach erhöht und in Abhängigkeit von vorliegender Depression als über 3-fach erhöht. Damit lässt sich am postinfektiösen Reizdarmsyndrom sehr gut der Zusammenhang von Hyperalgesie, Immunaktivierung und intestinaler Hyperalgesie belegen.

\section{Mikrobiom}

Dass der menschliche Darm von etwa 100 Billionen Keimen besiedelt ist, ist schon seit langem bekannt. Man betrachtete diese Keime lange Zeit als einfache Kommensalen, die die Reste unserer Nahrung verwerten, ohne dabei einen Einfluss auf unseren Körper zu nehmen. Erst die genetische Charakterisierung im 2007 gestarteten Human Microbiome Project in den USA [Koren et al., 2013] und im durch die Europäische Union (EU) finanzierten MetaHIT-Programm (Metagenomics of the Human Intestinal Tract) [Wylie et al., 2012; Arumugam et al., 2011] führte zu einem besseren Verständnis der Bedeutung der Darmbakterien für unseren Organismus und erlaubte eine bessere quantitative und qualitative Charakterisierung der bakteriellen Besiedelung. Dies führte zu einer explosionsartigen Vermehrung der Studien zum Mikrobiom von unter 100 pro Jahr bis 2001 auf nahezu 3000 im Jahr 2013. Die diesbezügliche Forschung ist noch jung und erlaubt keinen Gesamtüberblick. Es wird dennoch deutlich, dass sich der menschliche Organismus und die Darmbakterien in einer ständigen Interaktion befinden, in die unser Immunsystem, aber auch unsere Psyche involviert ist. Aus der Vielzahl der Studien seien nur wenige zitiert:

Sudo et al. [2004] verglichen Mäuse, die von Geburt an keimfrei gehalten wurden, mit Mäusen, die eine normale, pathogenfreie Darmflora entwickelten. Verglichen wurde die 
Reaktion auf einen typischen Stresstest bei Mäusen, bei denen die Extremitäten festgebunden werden. Die Reaktion der HPA-Achse, gemessen an der Freisetzung von adrenocorticotropem Hormon (ACTH) und Corticosteron, war dabei bei den keimfreien Mäusen sehr viel ausgeprägter als bei den normal keimbesiedelten Mäusen. Offensichtlich hat das Mikrobiom hier einen protektiven Einfluss gegen Stressfaktoren.

Bailey et al. [2011] verwendeten einen sozialen Stressor, indem Mäuse über $2 \mathrm{~h}$ hinweg in ihrem Bau einer aggressiven männlichen Maus ausgesetzt wurden. Dieser soziale Stressor führte zu einer signifikanten Veränderung der Darmflora, die bei den Kontrolltieren nicht beobachtet werden konnte. In unmittelbarem Zusammenhang mit der Änderung der Bakterienbesiedelung zeigte sich auch eine Erhöhung von zirkulierenden Entzündungsmediatoren. In einem Folgeexperiment wurde die Änderung der Darmflora durch Antibiotika unterdrückt. Unter diesen Bedingungen blieb der Anstieg der Entzündungsmediatoren aus, sodass ganz offensichtlich die Änderung der Darmflora tatsächlich für die Stressreaktion des Immunsystems verantwortlich war.

Der wissenschaftliche Diskurs hat mittlerweile den Bereich der gastrointestinalen Störungen und Erkrankungen so weit verlassen, dass die tiefgreifende Interaktion von Psyche, Gastrointestinaltrakt und Mikrobiom nur durch die Erweiterung der Brain-Gut-Achse zur Brain-Gut-Mikrobiom-Achse ausreichend verstanden werden kann. Das Mikrobiom beeinflusst bei Tieren das Verhalten, produziert selbst Neurotransmitter und nimmt direkten Einfluss auf die Hirnfunktion. Das Mikrobiom könnte auch eine bedeutsame Rolle für psychische Störungen wie die Depression spielen und die Wirkung von chronischem Stress moderieren [Dinan und Cryan, 2013]. Dies führt zu dem Versuch, psychische Störungen über eine direkte Einflussnahme auf das Mikrobiom durch die Ingestion von positiv wirkenden Mikroorganismen (die als «Psychobiotika» bezeichnet werden) zu behandeln [Dinan et al., 2013; Burnet und Cowen, 2013]. Was bei Tieren schon recht zuverlässig reproduzierbar ist, scheint auch beim Menschen von Bedeutung zu sein. Bei gesunden Menschen beeinflusste die Aufnahme von fermentierter Milch mit aktiven Mikroorganismen die Hirnaktivität in Ruhe und bei einer Aufgabe, bei der emotional Gesichter betrachtet werden sollten [Tillisch et al., 2013].

Welche Bedeutung diese tierexperimentellen Belege für die Interaktion von Mikrobiom, Immunsystem und Psyche für den Menschen haben, kann gegenwärtig noch nicht sicher abgeschätzt werden. Es ist jedoch zu erwarten, dass die Darmflora auch beim Menschen einen wichtigen modulierenden Effekt auf Stressreaktion, Immunantwort und Beschwerdeausprägung bei gastrointestinalen Störungen hat.

\section{Diskussion}

Die Psychophysiologie des Gastrointestinaltrakts hat sich von einfachen Grundannahmen zu einer hochkomplexen Be- trachtung der gesamten Brain-Gut-Achse weiterentwickelt. Am Anfang der wissenschaftlichen Beschäftigung mit den psychophysiologischen Zusammenhängen im Gastrointestinaltrakt standen sehr einfache Vorstellungen: Bestimmte Stressoren und emotionale Belastungen führen demgemäß zu interindividuell unterschiedlichen Funktionsänderungen, die dann eine typische Symptomausprägung bedingen. Man nahm an, dass die jeweilige emotionale Reaktion durch frühkindliche Erfahrungen geprägt und damit in erster Linie die Funktionsänderung gebahnt sei, in deren Folge dann auch die typische Symptomatik erklärt wäre.

Die Forschungsergebnisse stellten zunächst infrage, ob es denn überhaupt einen klaren Zusammenhang zwischen Symptomatik und Funktionsänderung gäbe. Soweit dies Motilitätsphänomene betraf, wurde zunehmend deutlich, dass sich zwar Motilitätsänderungen in Stresssituationen reproduzieren lassen, dass damit aber in aller Regel nicht gleichzeitig Symptome auftreten. Die Motilitätsphänomene reichen als Erklärung für das Auftreten der subjektiv beschriebenen Symptomatik nicht aus.

Gegenwärtig fehlt ein geschlossenes Modell für die Entstehung gastrointestinaler Beschwerden bei chronisch entzündlichen und funktionellen Magen-Darm-Erkrankungen. Die notwendigen Komponenten eines solchen Modells haben sich jedoch erheblich vermehrt. Stand ursprünglich nur die Motilität auf dem Prüfstand, hat sich bald erwiesen, dass die periphere Sensibilität eine wichtige Rolle spielt. In einem nächsten Forschungsschritt wurde deutlich, dass ein auf den Gastrointestinaltrakt bezogenes Verständnis der Hyperalgesie nicht ausreicht: Wesentlich sind offensichtlich auch die zentralnervöse Schmerzrezeption und die Einbeziehung unterschiedlicher Kerngebiete, die für die Stress- und Emotionsverarbeitung verantwortlich sind. Das bessere Verständnis der HPA-Achse mit der Differenzierung der CRF1- und CRF2-Rezeptoren zeigt, dass hierbei einerseits über die CRF2-Rezeptoren eine Hyposensibilität und andererseits über die CRF1-Rezeptoren eine Hyperalgesie vermittelt werden kann.

Auch bei den funktionellen Störungen des Magen-DarmTrakts spielen immunologische Vorgänge eine wichtige Rolle. Diese werden einerseits durch psychologische Faktoren, andererseits ebenfalls wieder über die HPA-Achse moderiert. Die immunologischen Vorgänge ihrerseits sind abhängig von der bakteriellen Darmbesiedelung, die wiederum von psychologischen Mechanismen abhängig ist.

Die notwendige Komplexität eines Modells, das den $\mathrm{Zu}$ sammenhang emotionaler Reaktionen mit gastrointestinalen Beschwerden erklären soll, hat sich durch die neuen Forschungsergebnisse ganz erheblich erhöht. Auf der anderen Seite sind aus theoretischen Vorstellungen, die sich eher an vorwissenschaftlichen Spekulationen orientiert haben, mittlerweile sehr gut belegte Einsichten in das Räderwerk der gastrointestinalen Physiologie geworden, deren Bedeutung für die gesamte Psychosomatik kaum überschätzt werden kann. 


\section{Disclosure Statement}

Der Autor erklärt hiermit, dass keinerlei Interessenskonflikte in Bezug auf dieses Manuskript bestehen.

\section{Literatur}

Alexander F: Psychosomatische Medizin: Grundlagen Gwee KA: Post-infectious irritable bowel syndrome, und Anwendungsgebiete, ed 4. Berlin, de Gruyter, 1985.

Almy TP, Kern F Jr, Abbot FK: Constipation and diarrhea, as reactions to life stress. Res Publ Assoc Res Nerv Ment Dis 1949;29:724-731.

-Almy TP: Experimental studies on the irritable colon. Am J Med 1951;10:60-67.

-Anderson KO, Dalton CB, Bradley LA, Richter JE: Stress induces alteration of esophageal pressures in healthy volunteers and non-cardiac chest pain patients. Dig Dis Sci 1989;34:83-91.

-Arumugam M, Raes J, Pelletier E, et al.: Enterotypes of the human gut microbiome. Nature 2011;473 174-180.

Ayres RC, Robertson DA, Naylor K, Smith CL: Stress and oesophageal motility in normal subjects and patients with irritable bowel syndrome. Gut 1989 ; 30:1540-1543

Bailey MT, Dowd SE, Galley JD, Hufnagle AR, Allen RG, Lyte M: Exposure to a social stressor alters the structure of the intestinal microbiota: implications for stressor-induced immunomodulation. Brain Behav Immun 2011;25:397-407.

Burnet PW, Cowen PJ: Psychobiotics highlight the pathways to happiness. Biol Psychiatry 2013;74: 708-709.

Connell AM: The motility of the pelvic colon. II. Paradoxical motility in diarrhoea and constipation. Gut 1962;3:342-348.

Dinan TG, Cryan JF: Melancholic microbes: a link between gut microbiota and depression? Neurogastroenterol Motil 2013;25:713-719.

Dinan TG, Stanton C, Cryan JF: Psychobiotics: a novel class of psychotropic. Biol Psychiatry 2013; 74:720-726.

-Elsenbruch S, Rosenberger C, Bingel U, Forsting M, Schedlowski M, Gizewski ER: Patients with irritable bowel syndrome have altered emotional modulation of neural responses to visceral stimuli. Gastroenterology 2010a;139:1310-1319.

-Elsenbruch S, Rosenberger C, Enck P, Forsting M, Schedlowski M, Gizewski ER: Affective disturbances modulate the neural processing of visceral pain stimuli in irritable bowel syndrome: an fMRI study. Gut 2010b;59:489-495.

Engel GL: Psychologic aspects of the management of patients with ulcerative colitis. N Y State J Med 1952;52:2255-2261.

Engel GL: Studies of ulcerative colitis. I. Clinical data bearing on the nature of the somatic process. Psychosom Med 1954a;16:496-501.

Engel GL: Studies of ulcerative colitis. II. The nature of the somatic processes and the adequacy of psychosomatic hypotheses. Am J Med 1954b;16:416433.

Gorard DA, Gomborone JE, Libby GW, Farthing MJ: Intestinal transit in anxiety and depression. Gut 1996;39:551-555.

Gwee KA, Leong YL, Graham C, et al.: The role of psychological and biological factors in postinfective gut dysfunction. Gut 1999;44:400-406. an inflammation-immunological model with relevance for other IBS and functional dyspepsia. J Neurogastroenterol Motil 2010;16:30-34.

Hislop IG: Psychological significance of the irritable colon syndrome. Gut 1971;12:452-457.

Johnson AC, Tran L, Schulkin J, Greenwood-Van Meerveld B: Importance of stress receptor-mediated mechanisms in the amygdala on visceral pain perception in an intrinsically anxious rat. Neurogastroenterol Motil 2012;24:479-486, e219.

Koren O, Knights D, Gonzalez A, et al.: A guide to enterotypes across the human body: meta-analysis of microbial community structures in human microbiome datasets. PLoS Comput Biol 2013;9: e1002863.

Larauche M, Mulak A, Tache Y: Stress and visceral pain: from animal models to clinical therapies. Exp Neurol 2012;233:49-67.

Latimer P: Psychophysiologic disorders: a critical appraisal of concept and theory illustrated with reference to the irritable bowel syndrome (IBS). Psychol Med 1979;9:71-80.

Mayer EA, Tillisch K: The brain-gut axis in abdominal pain syndromes. Annu Rev Med 2011;62:381-396.

Mertz H: Role of the brain and sensory pathways in gastrointestinal sensory disorders in humans. Gut 2002;51(suppl 1):i29-i33. of duodenal ulcer. I. Relation of specific psychological characteristics to rate of gastric secretion (serum pepsinogen). Psychosom Med 1957;19:1-10.

Pavlov VA, Tracey KJ: The vagus nerve and the inflammatory reflex - linking immunity and metabolism. Nat Rev Endocrinol 2012;8:743-754.

Rampton D: Does stress influence inflammatory bowel disease? The clinical data. Dig Dis 2009; 27(suppl 1):76-79.

Rao SS, Hatfield RA, Suls JM, Chamberlain MJ: Psychological and physical stress induce differential effects on human colonic motility. Am J Gastroenterol 1998;93:985-990.

Richter JE: Oesophageal motility disorders. Lancet 2001;358:823-828.

Ritchie J: Pain from distension of the pelvic colon by inflating a balloon in the irritable colon syndrome. Gut 1973;14:125-132.

Schmulson M, Lee OY, Chang L, Naliboff B, Mayer EA: Symptom differences in moderate to severe IBS patients based on predominant bowel habit. Am J Gastroenterol 1999;94:2929-2935.

Schmulson MJ: Brain-gut interaction in irritable bowel syndrome: new findings of a multicomponent disease model. Isr Med Assoc J 2001;3:104-110.

Schwille-Kiuntke J, Frick JS, Zanger P, Enck P: Postinfectious irritable bowel syndrome - a review of the literature. Z Gastroenterol 2011;49:997-1003.

Spiller R, Garsed K: Postinfectious irritable bowel syndrome. Gastroenterology 2009a;136:1979-1988.

Spiller R, Garsed K: Infection, inflammation, and the irritable bowel syndrome. Dig Liver Dis 2009b;41: 844-849.
Mirsky IA, Reiser MF, Thaler M, Weiner H: Etiology
Sudo N, Chida Y, Aiba Y, et al.: Postnatal microbial colonization programs the hypothalamic-pituitaryadrenal system for stress response in mice. J Physiol 2004;558(pt 1):263-275.

Tache Y, Martinez V, Million M, Maillot C: Role of corticotropin releasing factor receptor subtype 1 in stress-related functional colonic alterations: implications in irritable bowel syndrome. Eur J Surg Suppl 2002;587:16-22.

Tache Y, Martinez V, Wang L, Million M: CRF1 receptor signaling pathways are involved in stressrelated alterations of colonic function and viscerosensitivity: implications for irritable bowel syndrome. Br J Pharmacol 2004a;141:1321-1330.

Tache Y, Million M, Nelson AG, Lamy C, Wang L: Role of corticotropin-releasing factor pathways in stress-related alterations of colonic motor function and viscerosensibility in female rodents. Gend Med 2005;2:146-154.

Tache Y, Perdue MH: Role of peripheral CRF signalling pathways in stress-related alterations of gut motility and mucosal function. Neurogastroenterol Motil 2004b;16(suppl 1):137-142.

Tillisch K, Labus J, Kilpatrick L, et al.: Consumption of fermented milk product with probiotic modulates brain activity. Gastroenterology 2013;144: 1394-1401.

Tillisch K, Mayer EA, Labus JS: Quantitative metaanalysis identifies brain regions activated during rectal distension in irritable bowel syndrome. Gastroenterology 2011;140:91-100.

Wangel AG, Deller DJ: Intestinal motility in man. 3. Mechanisms of constipation and diarrhea with particular reference to the irritable colon syndrome. Gastroenterology 1965;48:69-84.

Welgan P, Meshkinpour H, Beeler M: Effect of anger on colon motor and myoelectric activity in irritable bowel syndrome. Gastroenterology 1988;94:11501156.

Welgan P, Meshkinpour H, Hoehler F: The effect of stress on colon motor and electrical activity in irritable bowel syndrome. Psychosom Med 1985;47: 139-149.

Welgan P, Meshkinpour H, Ma L: Role of anger in antral motor activity in irritable bowel syndrome. Dig Dis Sci 2000;45:248-251.

Whitehead WE, Holtkotter B, Enck P, et al.: Tolerance for rectosigmoid distention in irritable bowel syndrome. Gastroenterology 1990;98:1187-1192.

Whitehead WE, Palsson OS: Is rectal pain sensitivity a biological marker for irritable bowel syndrome: psychological influences on pain perception. Gastroenterology 1998;115:1263-1271.

Wylie KM, Truty RM, Sharpton TJ, et al.: Novel bacterial taxa in the human microbiome. PLoS One 2012; 7:e35294.

Yuan PQ, Wu SV, Elliott J, et al.: Expression of corticotropin releasing factor receptor type 1 (CRF1) in the human gastrointestinal tract and upregulation in the colonic mucosa in patients with ulcerative colitis. Peptides 2012;38:62-69. 